\title{
Monetary Policy with Asset-Backed Money
}

\author{
David Andolfatto, \\ Aleksander Berentsen \\ and \\ Christopher J. Waller
}

\author{
Working Paper 2013-030A \\ https://doi.org/10.20955/wp.2013.030
}

October 2013

\author{
FEDERAL RESERVE BANK OF ST. LOUIS \\ Research Division \\ P.O. Box 442 \\ St. Louis, MO 63166
}

The views expressed are those of the individual authors and do not necessarily reflect official positions of the Federal Reserve Bank of St. Louis, the Federal Reserve System, or the Board of Governors.

Federal Reserve Bank of St. Louis Working Papers are preliminary materials circulated to stimulate discussion and critical comment. References in publications to Federal Reserve Bank of St. Louis Working Papers (other than an acknowledgment that the writer has had access to unpublished material) should be cleared with the author or authors. 


\title{
Monetary Policy with Asset-Backed Money
}

\author{
D. Andolfatto \\ Federal Reserve Bank of St. Louis \\ A. Berentsen \\ University of Basel and Federal Reserve Bank of St. Louis \\ C. Waller \\ Federal Reserve Bank of St. Louis
}

August 2013

\begin{abstract}
We study the use of intermediated assets as media of exchange in a neoclassical growth model. An intermediary is delegated control over productive capital and finances itself by issuing claims against the revenue generated by its operations. Unlike physical capital, intermediated claims are assumed to be liquid-they constitute a form of asset-backed money. The intermediary is assumed to control 1) the number of claims outstanding, 2) the dividends paid out to claim holders and 3 ) the fee charged for collecting the dividend. We find that for patient economies, the first-best allocation can always be implemented as a competitive equilibrium through an appropriately designed intermediary policy rule. The optimal policy requires strictly positive inflation. While it is also possible to implement the first-best by introducing fiat money and a lumpsum tax instrument, our results demonstrate that neither of these interventions are necessary for efficiency.
\end{abstract}

\section{Introduction}

The end of Bretton Woods in 1971 ushered in the era of fiat currencies. This decoupling of currency from a commodity standard raised many issues among economists such as price level determinacy, the optimal rate of inflation, and most importantly, who should be in charge of the monetary system - the government or the private sector? Friedman (1969), Klein (1974, 1976) and Hayak (1976) argued strenuously that privately managed monetary arrangements were feasible and would lead to the best economic outcomes. Under this system a commodity backed private currency, 
in conjuction with competitive note issuance, would pave the way to price stability, i.e., zero inflation. The main point of contention was whether or not a government provided monopoly provided fiduciary currency was "essential" in such a system. In short, is there something special about government fiat currency?

The inflation of the 1970's rekindled this debate in the 1980's, with work by Barro (1979), King (1983), Wallace (1983), Sargent and Wallace (1983) and Friedman and Schwartz (1986). Again, the focus on private monetary sytems focused on commodity money backed by gold or silver. However, Fama (1983) argued that claims backed by a portfolio of assets was sufficient and actually had better properties than a specie backed currency. Under his arrangment, the financial intermediary did not issue liabilities redeemable in specia since the claims were equity claims. The financial intermediary was simply a conduit for passing along the returns on the underlying assets to the claim holders. Nevertheless, Fama argues that due to information and computation costs, fiat currency would still be needed for "hand to hand" transactions. While the Great Moderation and the decline in worldwide inflation since the early 1980's caused the profession to lose interest in this topic, the recent financial crisis has led to a renewed public debate on the necessity of government fiduciary currency, most notably from the "End the Fed" crowd in the U.S..

Although the literature on privately managed monetary systems focuses on many dynamic issues such as price stability, surprisingly, none of this work was done using choice theoretic, dynamic general equilibrium models. ${ }^{1}$ Much of the analysis is either static, purely intuitive or focuses on historical episodes. Also problematic is that the underlying frictions giving rise to the need for currency were not well specified. This was an obvious problem that was recognized early on as evidenced by Helpman's (1983) discussion of Fama's paper:

"The argument for an uncontrolled banking system is made on efficiency grounds by means of the frictionless neoclassical model of resource allocation. But this framework does not provide a basis for arguing the desirability of price level stabilization. If indeed stabilization of the price level is desirable, we need to know precisely what features of the economy lead to it. Then we have to examine whether such features make an uncontrolled banking system desirable. This problem is of major importance, but it is not addressed in the paper." (p. 30).

However, due to advances in macroeconomic and monetary theory, we are now able to use choice theoretic, dynamic general equilibrium models to revisit these issues. Modern monetary theory has made clear progress in addressing Helpman's critique of Fama's work by specifying the frictions needed to make a medium of

\footnotetext{
${ }^{1}$ A notable exception is Sargent and Wallace (1983) which studies a commodity money economy in an overlapping generations framework.
} 
exchange essential for trade. These frictions include, a lack of record-keeping (public communication of individual trading histories) and a lack of commitment. It is often claimed that these frictions make fiat money essential. However, in papers making this claim, no other asset exists to serve as medium of exchange. In fact, when a second asset exists, rate of return dominance typically leads to fiat money being discarded as a medium of exchange unless the Friedman rule is implemented.

To address this point, a body of research developed that studied the conditions under which fiat money and other assets could coexist as media of exchange. One strand of this literature shows that if a real asset can be used as media of exchange and its fundamental value is sufficiently high, then the first-best allocation can be obtained. However, if the fundamental value is too low, the real asset will carry a liquidity premium and the first best allocation is unattainable [see Waller (2003), Geromichalos et al (2007) and Lagos and Rocheteau (2008)]. ${ }^{2}$ It then follows that introducing a second asset (typically fiat money) to reduce this liquidity premium will lead to better allocations. Thus, fiat money appears to be essential even if real assets are available as exchange media.

While intuitive, this explanation is puzzling for the following reason. The stock of fiat money can be interpreted as claims to a real asset with a zero dividend - think of claims to a Lucas tree that bears no fruit. Despite having no fundamental value, policies still can be constructed such that the first-best allocation is achieved with these claims, e.g., implement the Friedman rule by contracting the money supply at the rate of time preference. But if this can be done for a zero dividend asset, then it should be feasible to do the same for assets with positive dividends. This suggests that, contrary to earlier claims, a second medium of exchange is not needed. What is needed is an appropriately designed policy for the asset-backed money supply. One objective of our paper is to show that if the supply of asset-backed money is managed in an optimal fashion, then fiat money is not essential. A second objective is to demonstrate precisely what an optimal policy looks like.

We use the Aruoba and Wright (2003) neoclassical growth model to study the use of intermediated assets as media of exchange. An intermediary is delegated control over productive capital and finances itself by issuing claims against the revenue generated by its operations. ${ }^{3}$ Unlike physical capital, intermediated claims are assumed to be liquid-they constitute a form of asset-backed money. The intermediary is assumed to control 1) the number of claims outstanding, 2) the dividends paid out to claim holders and 3) the fee charged for collecting the dividend. We find that for pa-

\footnotetext{
${ }^{2}$ Another strand of this literature assumes there is an asymmetric information problem with the real assets [Rocheteau (2011) and Lester et al (2012)]. A different approach is that of Jacquet and Tan (2012) who assume that the real asset has better consumption hedging properties for some agents than others.

${ }^{3}$ Will Roberds has pointed out to us that the central banks of 300-600 years ago operated exactly in this manner. See Fratianni (2006).
} 
tient economies, the first-best allocation can always be implemented as a competitive equilibrium through an appropriately designed intermediary policy rule. The optimal policy requires a growing nominal supply of asset-backed money-some strictly positive inflation is optimal. While it is also possible to implement the first-best by introducing fiat money and a lump-sum tax instrument, our results demonstrate that neither of these interventions are necessary to ensure the efficient management of the economy's money supply.

We organize our paper as follows. In Section 2, we describe the environment and characterize the first-best allocation. In section 3, we describe the competitive equilibrium of our model economy assuming that 1) agents are anonymous (recordkeeping and commitment are absent), 2) an intermediary is delegated control over the physical capital stock, with its intermediated claims serving as an exchange medium, and 3) the intermediary follows an exogenous policy rule that governs the distribution of dividends, the retention of earnings, and the nominal supply of its liabilities. In section 4, we characterize a stationary monetary equilibrium under the assumption of zero intervention-a policy that keeps the nominal quantity of liabilities constant and collects zero revenue, apart from net capital income. In section 5, we describe the properties of an optimal policy and discuss our findings. Section 6 concludes.

\section{Environment}

The environment is that of Aruoba and Wright (2003). ${ }^{4}$ Time $t$ is discrete and the horizon is infinite. Each period is divided into two subperiods, which we refer to below as the AM and PM (subperiods), respectively. There is a $[0,1]$ mass of ex ante identical agents with preferences:

$$
E_{0} \sum_{t=0}^{\infty} \beta^{t}\left\{\sigma\left[u\left(c_{t}\right)-q_{t}\right]+\theta U\left(C_{t}\right)-N_{t}\right\}
$$

Agents are randomly assigned to one of three states in the AM: consumers, producers, or idlers. The probability of becoming a consumer is $\sigma$, the probability of becoming a producer is $\sigma$, and the probability of becoming an idler is $1-2 \sigma$, where $0<\sigma<1 / 2$. The AM good is nonstorable. A consumer derives flow utility $u\left(c_{t}\right)$ from consuming the AM good $c_{t}$ and a producer derives flow utility $-q_{t}$ from producing the $\mathrm{AM}$ good $q_{t}$ at time $t$. The AM flow utility for idlers is normalized to zero. Assume $u^{\prime \prime}<0<u^{\prime}$ and $u(0)=0, u^{\prime}(0)=\infty$. As there is an equal mass of producers and consumers, feasibility and efficiency imply $c_{t}=q_{t}$.

All agents have the same preferences and opportunities in the PM. Their PM flow utility payoff is given by $\theta U\left(C_{t}\right)-N_{t}$, where $C_{t}$ is consumption of the PM good and

\footnotetext{
${ }^{4}$ Aruoba and Wright (2003) introduce illiquid capital into the Lagos and Wright (2005) framework.
} 
$N_{t}$ denotes labor effort at time $t$. Assume that $U^{\prime \prime}<0<U^{\prime}$ with $U(0)=-\infty$ and $U^{\prime}(0)=\infty$. The parameter $\theta$ indexes the relative weight agents place on consumption vis-à-vis labor in their preferences and will play an important role below. Production of the PM output is standard neoclassical: $Q_{t}=F\left(K_{t}, N_{t}\right)$, where $F$ exhibits all the usual properties. Let $f(k) \equiv F(K / N, 1)$, where $k \equiv K / N$. The PM output is storable, so the resource constraint is given by:

$$
C_{t}=F\left(K_{t}, N_{t}\right)+(1-\delta) K_{t}-K_{t+1}
$$

with $K_{0}>0$ given. In what follows, we restrict attention to stationary allocations. The first-best allocation $\left(q^{*}, k^{*}, C^{*}, K^{*}\right)$ satisfies:

$$
\begin{aligned}
u^{\prime}\left(q^{*}\right) & =1 \\
\beta\left[f^{\prime}\left(k^{*}\right)+1-\delta\right] & =1 \\
{\left[f\left(k^{*}\right)-f^{\prime}\left(k^{*}\right) k^{*}\right] \theta U^{\prime}\left(C^{*}\right) } & =1 \\
{\left[f\left(k^{*}\right) / k^{*}-\delta\right] K^{*} } & =C^{*}
\end{aligned}
$$

Lemma $1 q^{*}$ and $k^{*}$ are determined independently of $\theta . K^{*}(\theta), N^{*}(\theta)$ and $C^{*}(\theta)$ are strictly increasing in $\theta$.

Aruoba and Wright (2003) examine the properties of equilibria under the assumptions that: (i) agents are anonymous, (ii) capital cannot be used as a payment instrument in the AM market; and (iii) there is a fiat money instrument that can be used as a payment instrument in the AM market. They find that the Friedman rule (deflate at the rate of time preference) is an optimal policy. ${ }^{5}$

In this paper, we maintain assumptions (i) and (ii) used in Aruoba and Wright (2003), but we replace their assumption (iii) with an arguably weaker restriction. That is, instead of a fiat or intermediary debt instrument, we assume that money can take the form of an intermediated claim on capital income (and potentially other income sources). We refer to this intermediated claim as asset-backed-money (ABM). We view this restriction as weaker in the sense that fiat money is just a limiting case of ABM for which backing is entirely absent. Our purpose is to examine the properties of an optimal monetary policy when the economy's payment instrument takes the form of an intermediated asset-backed security.

\footnotetext{
${ }^{5}$ Because of their search and bargaining friction, the Friedman rule may not implement the firstbest allocation. But it remains the best that policy can accomplish by way of inflation policy.
} 


\section{An asset-backed-money economy}

\subsection{Markets and prices}

All markets are competitive. ${ }^{6}$ Factor market equilibrium in the PM implies that capital and labor earn their respective marginal products. Consequently, the PM real wage and rental rates respectively satisfy:

$$
\begin{aligned}
w(k) & =f(k)-f^{\prime}(k) k \\
r(k) & =f^{\prime}(k)
\end{aligned}
$$

where for convenience, we heretofore drop the time subscripts and let $\left(x^{-}, x, x^{+}\right)$ denote the prior-period, current-period, and next period value of variable $x$, respectively. There are also competitive spot markets in the AM and PM, where ABM is exchanged for output. Let $\left(\phi_{1}, \phi_{2}\right)$ denote the price of these securities measured in units of AM and PM output, respectively.

\subsection{The intermediary}

We assume the existence of an agency programmed to act like an intermediary that transforms illiquid capital into a payment instrument-ABM. Some readers may prefer to think of our intermediary as an exogenous government policy rule. But nothing in our description of the intermediary requires such an interpretation. In fact, we prefer to think of the agency as a large private bank with covenants designed to maximize membership welfare. Importantly, membership is optional in our model (we do not assume the existence of a lump-sum tax instrument).

As physical capital is assumed to be illiquid in the hands of private agents, it can be shown that agents would prefer to hold indirect (liquid) claims to capital issued by the intermediary. Thus, we assume that the intermediary has direct ownership of the physical capital stock. The initial quantity of capital is acquired by issuing liabilities (durable, divisible, noncounterfeitable) representing claims against the stream of income generated by the intermediary's business activities. New capital investment is financed by a combination of revenue sources available to the intermediary, which we now make explicit by stating the intermediary's budget constraint:

$$
D=r(k) K+\phi_{2}\left(M^{+}-M\right)+T+(1-\delta) K-K^{+}
$$

with $K_{0}>0$ given.

Let us now describe the objects in (7). $D$ represents the aggregate real dividend paid out by the intermediary in the PM. $r K$ represents capital income the intermediary earns by renting its capital in the PM factor market. $\phi_{2}\left(M^{+}-M\right)$ represents

\footnotetext{
${ }^{6}$ Bargaining frictions play no essential role in our analysis, so we abstract from them for simplicity.
} 
real seigniorage revenue, and $T$ represents revenue collected from voluntary (sequentially rational) contributions that resemble redemption fees. Finally, $K^{+}-(1-\delta) K$

represents provisions made for new capital investment. In a stationary state, the intermediary's budget constraint satisfies:

$$
D=(r(k)-\delta) K+(\mu-1) \phi_{2} M+T
$$

where $\mu=M^{+} / M$.

The intermediary's behavior is described by a set of exogenous policy rules. So, for example, under zero intervention we set $\mu=1$ and $T=0$, so that $D=(r-\delta) K$. In general, given any $(\mu, T)$, a feasible policy rule must satisfy (8).

Define $d \equiv D / M$; that is, the real dividend per unit of ABM. An individual who enters the PM with $m$ units of ABM is entitled to receive $m d$ units of PM output (and retain the ex-dividend value of their $\mathrm{ABM} \phi_{2} m$ ), but only if the bearer pays a real redemption fee equal to $\tau$ units of PM output. In a stationary equilibrium, $D=d M$ is constant. This implies that if $\mu>(<) 1$, then dividend paid per unit of money $d$ declines (rises) in a stationary equilibrium.

\subsection{Individual decision-making}

\subsubsection{The PM market}

An individual who enters the PM with $m$ units of ABM faces the budget constraint:

$$
c+\phi_{2} m^{+}=\left(\phi_{2}+\chi d\right) m+w n-\chi \tau
$$

where $m^{+} \geq 0$ denote the notes carried into the "next period" AM, $n$ represents individual labor supply, and $\tau$ is a redemption fee, and $\chi \in\{0,1\}$ denotes the redemption choice. The act of redemption is sequentially rational if and only if:

$$
d m \geq \tau
$$

This, in turn, implies that only agents entering the PM market with large enough money balances $(m \geq \tau / d)$ can be induced to make a voluntary contribution. Since the redemption choice is static in nature, we may without loss take it as exogenous for the time being. The choice will be modeled at the appropriate place below when we discuss incentive-feasible allocations.

In what follows, we conjecture (and later verify) that the PM redemption option is exercised by those who were producers and idle agents in the previous AM market. Consumers in the previous AM will have depleted their ABM balances to a point that makes redemption suboptimal. 
Associated with the asset positions $\left(m, m^{+}\right)$are the value functions $W(m)$ and $V\left(m^{+}\right)$which must satisfy the following recursive relationship:

$$
W(m) \equiv \max \left\{\theta U(c)-n+\beta V\left(m^{+}\right): c=\left(\phi_{2}+\chi d\right) m+w n-\phi_{2} m^{+}-\chi \tau\right\}
$$

Assuming that $V$ is strictly concave (a condition that can be shown to hold in the relevant range of parameters we consider below), we have the following first-order conditions describing optimal behavior:

$$
\begin{aligned}
1 & =w \theta U^{\prime}(c) \\
\phi_{2} & =w \beta V^{\prime}\left(m^{+}\right)
\end{aligned}
$$

By the envelope theorem,

$$
W^{\prime}(m)=(1 / w)\left(\phi_{2}+\chi d\right)
$$

\subsubsection{The AM market}

A consumer that enters the AM with $m$ units of money faces the following problem:

$$
V_{c}(m) \equiv \max \left\{u(q)+W\left(m^{\prime}\right): m^{\prime}=m-\phi_{1}^{-1} q \geq 0\right\}
$$

Let $\lambda$ denote the Lagrange multiplier associated with the non-negativity constraint $m^{\prime} \geq 0$. Then the optimality condition is given by:

$$
\phi_{1} u^{\prime}(q)=(1 / w)\left(\phi_{2}+\chi d\right)+\lambda
$$

where here, we make use of (14). If the constraint is slack, then $\lambda=0$ and

$$
\phi_{1} m \geq q
$$

Otherwise, the constraint binds so that $\phi_{1} m=q$. By the envelope theorem:

$$
V_{c}^{\prime}(m)=\phi_{1} u^{\prime}(q)
$$

A producer that enters the AM with $m$ units of money faces the following problem:

$$
V_{p}(m) \equiv \max \left\{-q+W\left(m^{\prime}\right): m^{\prime}=m+\phi_{1}^{-1} q\right\}
$$

In equilibrium, the following must hold:

$$
\phi_{1}=(1 / w)\left(\phi_{2}+\chi d\right)
$$

where here, we make use of (14). By the envelope theorem:

$$
V_{p}^{\prime}(m)=\phi_{1}
$$

Finally, for the idle agents who enter with $m$ units of money, $V_{i}(m)=W(m)$, so that:

$$
V_{i}^{\prime}(m)=\phi_{1}
$$

where here again we make use of (14). 


\subsection{Stationary monetary equilibrium}

We focus on symmetric stationary equilibria. Such equilibria meet the following requirements: (i) Households' decisions are optimal, given prices and policy; (ii) The decisions are symmetric across all sellers and symmetric across all buyers; (iii) Markets clear at every date; (iv) All real quantities are constant across time; and (v) The intermediary budget constraint (8) holds in each period.

Let us gather the restrictions implied by behavior. Using (17), (19) and (20), allows us to form: $V^{\prime}(m)=\sigma V_{c}^{\prime}(m)+\sigma V_{p}^{\prime}(m)+(1-2 \sigma) V_{i}^{\prime}(m)$ which, when combined with condition (13) yields $\phi_{2}=w^{+} \beta\left\{\sigma \phi_{1}^{+} u^{\prime}\left(q^{+}\right)+\sigma \phi_{1}^{+}+(1-2 \sigma) \phi_{1}^{+}\right\}$. Backdating this latter expression by one period and collecting terms, we have:

$$
\phi_{2}^{-}=w \beta L(q) \phi_{1}
$$

where $L(q) \equiv \sigma\left[u^{\prime}(q)-1\right]+1$. Note that $L^{\prime}(q)<0$ and $L\left(q^{*}\right)=1$.

Note that in a stationary equilibrium, the aggregate real value of outstanding claims is constant: $\phi_{2}^{-} M^{-}=\phi_{2} M$. Since $M=\mu M^{-}$, it follows that $\phi_{2}^{-}=\mu \phi_{2}$. Combine this latter expression with (21) and multiply both sides by $M$ to derive:

$$
\mu \phi_{2} M=w \beta L(q) \phi_{1} M
$$

In a similar vein, multiply both sides of condition (18) by $M$ to derive:

$$
\phi_{1} M=(1 / w)\left(\phi_{2} M+D\right)
$$

where, recall $D=d M$.

Next, from the consumers' choice problem, the constraint $\phi_{1} m \geq q$ either binds or is slack. If the value function $V(m)$ is strictly concave, then quasilinear preferences imply that all agents enter the AM with identical money holdings. If $V(m)$ is linear, then our assumption of symmetry implies (without loss) the same thing. Marketclearing therefore implies $m=M$ at every date, so that (16) implies:

$$
\phi_{1} M \geq q
$$

To close the model, we impose the condition that in real terms, the outstanding ABM supply $M$ is priced at its liquidation value in the PM market; i.e.,

$$
\phi_{2} M=K
$$

Condition (25) corresponds to Tobin's $Q$ relation. We assume that if (25) does not hold, then intermediary is programmed to exploit a resulting arbitrage opportunity. In particular, if $\phi_{2} M>K$, the intermediary issues additional claims and sells them for PM output (the proceeds of which may be distributed or destroyed) whilst keeping the operating capital stock $K$ and business intact. Similarly, if $\phi_{2} M<K$, the 
intermediary threatens to liquidate its capital stock, making a profit $K-\phi_{2} M>0$ (which again may be distributed or destroyed), and then restarting its business by issuing new claims worth $\phi_{2} M=K^{\prime}<K$.

Combine (25) with (22) and (23) to get:

$$
\mu K=w(k) \beta L(q) \phi_{1} M
$$

and

$$
\phi_{1} M=[1 / w(k)](K+D)
$$

Define $s \equiv T / K$, and combine (25) and (6) with the intermediary budget constraint (8) to get:

$$
D=\left[f^{\prime}(k)-\delta+\mu+s-1\right] K
$$

And from condition (12) we have:

$$
w(k) \theta U^{\prime}([f(k) / k-\delta] K)=1
$$

where $C=[f(k) / k-\delta] K$. In addition, we have condition (24), which implies:

$$
\phi_{1} M \geq q^{*} \text { or } \phi_{1} M=q<q^{*}
$$

Conditions (26)-(30) characterize the stationary equilibrium values $\left(q, k, K, D, \phi_{1}\right)$ under the assumption that redemption choices are given and consistent with budget balance for the intermediary. We now need to state the conditions that guarantee this will be the case.

We know that strictly away from the first-best allocation, the equilibrium distribution of money at the beginning of the PM is as follows: consumers hold zero money, idlers hold $M$ units of money, and producers hold $2 M$ units of money. This distribution of money continues to be an equilibrium as we approach the first-best allocation. We restrict attention here to incentive schemes that satisfies $d M \geq \tau$, so that producers strictly prefer to exercise the redemption option while idle agents weakly prefer to do so.

In this case, total contributions are $T=(1-\sigma) \tau$. Recall from (28) that $D=$ $\left[f^{\prime}(k)-\delta+\mu+s-1\right] K$, so that (10) and $m=M$ implies $D \geq \tau$, so that:

$$
\left[f^{\prime}(k)-\delta+\mu+s-1\right] K \geq(1-\sigma)^{-1} T
$$

Recall that $s=T / K$ so that the expression above implies:

$$
\left[f^{\prime}(k)-\delta+\mu+s-1\right] \geq(1-\sigma)^{-1} s
$$

For a given $k$, condition (31) defines a class of policies $(\mu, s)$ consistent with voluntary contributions of $\tau$ among the producers and idlers. As we shall see below, this condition may not be satisfied at the first-best allocation for some economies. 
Finally, since agents lack commitment (implicit in our assumption of anonymity), the proposed allocation must be sequentially rational. This requirement is automatically satisfied as all markets are competitive (people are free to produce or work zero amounts at any point in time). There is a question as to whether sequential rationality prevents first-best implementation. In general, the answer is yes (typically, for low $\beta$ economies). In the present context, however, the answer is no because of our assumption on preferences: $U(0)=-\infty$. In short, "opting out" is infinitely painful for all $\beta$ so that the threat of opting out can be ignored.

\section{Zero intervention}

In this section, we characterize the stationary monetary equilibrium under zero intervention: $\mu=1$ and $s=0$. In this case, $\chi=1$ for all agents and condition (31) can be ignored. From (28), this implies that the aggregate real dividend is equal to capital income net of depreciation expense; i.e., $D=\left[f^{\prime}(k)-\delta\right] K$. It also implies that asset prices are constant over time. The question that concerns us here is whether the constraint (30) binds or not. To begin, we first assume that the constraint is slack and then verify that this will be the case in a certain region of the parameter space.

\subsection{Case 1: Debt constraint slack}

Combine (27) and (28) to form:

$$
\phi_{1} M=(1 / w(k))\left(1+f^{\prime}(k)-\delta\right) K
$$

Use (26) to get $w(k)=K /\left(\beta \phi_{1} M\right)$ and combine with the expression above:

$$
\phi_{1} M=\frac{\beta \phi_{1} M}{K}\left(1+f^{\prime}(k)-\delta\right) K
$$

or

$$
1=\beta\left(1+f^{\prime}\left(k^{*}\right)-\delta\right)
$$

That is, if $\phi_{1} M \geq q^{*}$, then $k=k^{*}$ and the equilibrium is first-best; see (2).

Now, from (26) we have $\phi_{1} M=K^{*} /\left(w\left(k^{*}\right) \beta\right)$. Combining this with (30) implies:

$$
K^{*}(\theta)>q^{*} w\left(k^{*}\right) \beta
$$

Lemma 2 There exists a unique $0<\theta_{0}<\infty$ satisfying $K^{*}\left(\theta_{0}\right)=q^{*} w\left(k^{*}\right) \beta$. For economies with $\theta \geq \theta_{0}$, the competitive monetary equilibrium is efficient under zero intervention. 
Lemma 2 follows from the fact that $K^{*}(\theta)$ is strictly increasing in $\theta$ and that $\left(q^{*}, k^{*}\right)$ are independent of $\theta$; i.e., see Lemma 1 . For the case of zero intervention, the choice $\chi=1$ is obviously optimal for all agents and satisfies budget balance.

We now move to characterize the competitive equilibrium under zero intervention for economies in which $0<\theta<\theta_{0}$.

\subsection{Case 2: Debt constraint tight}

From condition (26) and the definition of $\theta_{0}$ in Lemma 2, we know:

$$
\phi_{1} M=K^{*}\left(\theta_{0}\right) /\left(w\left(k^{*}\right) \beta\right)=q^{*}
$$

Since $\theta<\theta_{0}$, Lemma 1 implies that $K^{*}(\theta)<K^{*}\left(\theta_{0}\right)$, which implies $\phi_{1} M<q^{*}$ so that $\phi_{1} M=q<q^{*}$.

Use (26) to form $w(k)=K /\left(\beta L(q) \phi_{1} M\right)$ and combine with (32) to get:

$$
1=\beta L(q)\left(1+f^{\prime}(k)-\delta\right)
$$

Since $q<q^{*}$, we have $L(q)>1$ so that the condition above implies $k>k^{*}$. Condition (29) then implies $K(\theta)>K^{*}(\theta)$; that is, capital is overaccumulated relative to the first-best allocation.

The key results here are summarized by the following lemma:

Lemma 3 For economies with $0<\theta<\theta_{0}$, the competitive monetary equilibrium is inefficient under zero intervention. Moreover:

$$
\begin{aligned}
& \phi_{1} M=q<q^{*} \\
& \phi_{2} M=K>K^{*}
\end{aligned}
$$

That is, in a constrained-equilibrium, the AM price of money is "too low" and the PM price of money is "too high." Relatedly, the rate of return on money (and capital) is too low (there is a liquidity premium):

$$
\frac{1}{\beta L(q)}=\left(1+f^{\prime}(k)-\delta\right)=\left[\frac{\phi_{2}+d}{\phi_{2}}\right]<\frac{1}{\beta}
$$

\section{Optimal policy}

For sufficiently low values of $\theta$ the first best is not attainable with a passive policy. The question that remains to be determined is whether it can be with an appropriate intervention and, if so, what this intervention looks like. We can now state the key proposition of our paper. 
Proposition 4 When $0<\theta<\theta_{0}$, there exists $0<\beta_{0}<1$ for which the first-best allocation can be implemented as a competitive monetary equilibrium with a policy that sets $\mu>1$ and $s>0$, for any $\beta \geq \beta_{0}$.

Proof. Since we have $\theta<\theta_{0}$, Lemma 1 implies $K^{*}(\theta)<K^{*}\left(\theta_{0}\right)$. From Lemma $2, K^{*}\left(\theta_{0}\right)=q^{*} w\left(k^{*}\right) \beta$. At the first-best allocation, the equilibrium capital stock $K(\theta)=K^{*}(\theta)$. Condition (26) places the following restriction on the equilibrium capital stock:

$$
\mu K^{*}(\theta)=w\left(k^{*}\right) \beta q^{*}=K^{*}\left(\theta_{0}\right)
$$

Hence, first-best implementation requires $\mu=K^{*}\left(\theta_{0}\right) / K^{*}(\theta)>1$. Next, combine (26), (27) and (28) to form $\mu=\beta\left(f^{\prime}\left(k^{*}\right)-\delta+\mu+s\right)$. Since $f^{\prime}\left(k^{*}\right)-\delta=1 / \beta-1$, we have:

$$
s=\left(\frac{1-\beta}{\beta}\right)(\mu-1)
$$

An optimal intervention $\left(\mu^{\prime}, s^{\prime}\right)$ satisfies $\mu^{\prime} \geq \mu, s^{\prime} \geq s$, where $(\mu, s)$ satisfy conditions (36) and (37).

Finally, replace $f^{\prime}\left(k^{*}\right)-\delta=(1-\beta) / \beta$ in condition (31) to derive:

$$
\left[\frac{1-\beta}{\beta}+\mu+s-1\right] \geq(1-\sigma)^{-1} s
$$

For $\mu$ defined in (36), combine (37) and (38) to derive:

$$
1 \geq\left[\left(\frac{\sigma}{1-\sigma}\right)-\left(\frac{\beta}{1-\beta}\right)\right](\mu-1)
$$

Condition (39) implies that $\beta \geq \beta_{0}=\sigma$ is a sufficient condition (recall that $\sigma<1 / 2$ ) for an optimal policy to induce the requisite voluntary contributions.

\subsection{Discussion}

Aruoba and Wright (2003) assume that physical capital is illiquid in the sense that capital cannot be used as a payment instrument in the AM market. To facilitate trade in that market, a new asset is introduced-a fiat money object (more generally, government debt) that is assumed to be liquid. As is standard, they find that the Friedman rule $(\mu=\beta<1)$ is an optimal policy. That is, if lump-sum taxation is available, then it should be used to contract (deflate) the money supply to generate an efficient real rate of return on money.

Lagos and Rocheteau (2008) modify the Aruoba and Wright (2003) model by permitting capital to circulate as a payment instrument. In some cases, capital is overaccumulated-a result that is familiar in overlapping generations models and, indeed, in the model we consider here. When this is so, the introduction of a second 
asset-again, fiat money or government debt-can improve efficiency. The optimal policy is again the Friedman rule $(\mu=\beta<1)$.

Our approach contrasts with the previous two papers in the follow ways. First, we assume that capital is illiquid, but that intermediated claims to capital are not. Second, we do not (although we could) introduce a second asset (like fiat money or government debt). Consequently, monetary policy in our model is restricted to managing the supply of intermediated claims-ABM. We find that an optimal policy requires inflation-which is equivalent to the intermediary following a policy of persistent (and predictable) share dilution. Third, implementation in our model is possible even without lump-sum taxes, at least, in patient economies.

The result that a strictly positive inflation is optimal is, at first blush, somewhat puzzling. In a large class of monetary models, the Friedman rule is an optimal policy. In models where inflation is desirable, there is usually a redistributive motive at work; see, for example, Levine (1991) and Molico (2006). Because of our restriction to quasilinear preferences, that redistributive motive is absent here. Andolfatto (2010) argues that deflation is necessarily ruled out for any policy that induces voluntary tax payments. However, our policy prescription here continues to hold even when we interpret $\tau$ as a lump-sum tax. So clearly, there must be something else going on here.

To develop some intuition for our result, consider the following argument. Note that (34) and (35), together with Lemma 2, imply that there is a $\theta_{0}$ for which the following is true:

$$
\frac{\phi_{2}}{\phi_{1}}=\frac{K^{*}\left(\theta_{0}\right)}{q^{*}}
$$

For $\theta<\theta_{0}$, the economy does not desire the PM consumption good as much, leading to a decline in the quantity of capital required to produce it. That is

$$
\frac{\phi_{2}}{\phi_{1}}=\frac{K(\theta)}{q}>\frac{K^{*}(\theta)}{q^{*}}
$$

To restore efficiency, we want $\phi_{2}$ to decline with $\theta$. But the value of these ABM claims may not be sufficient to purchase the efficient level of AM output. As a result, capital is overaccumulated (relative to the first-best) and AM production falls below its first-best level. This situation creates the price distortion described in Lemma 3; i.e., $\left(\phi_{2} / \phi_{1}\right)$ is too high.

Now, consider the pricing equation (26). At the first-best, this equation implies:

$$
\mu\left(\frac{\phi_{2}}{\phi_{1}}\right)=\beta w\left(k^{*}\right)
$$

Equations (40) and (41) imply that the only way to reduce $\phi_{2}$ and keep $\phi_{1}$ fixed as $\theta$ 
declines below $\theta_{0}$ is to set the inflation rate in a manner that satisfies:

$$
\mu(\theta)\left(\frac{K^{*}(\theta)}{q^{*}}\right)=\beta w\left(k^{*}\right)
$$

Condition (42), which derived by combining (40) and (41), implies that the efficient inflation rate $\mu(\theta)$ is decreasing in $\theta$, for $\theta<\theta_{0}$. Since $\mu\left(\theta_{0}\right)=1$ (zero inflation is optimal when $\left.\theta \geq \theta_{0}\right)$, it follows that $\mu(\theta)>1$ for $\theta<\theta_{0}$. By printing nominal claims at a faster pace, the purchasing power of ABM in the PM market is reduced. The effect here is to mitigate the overaccumulation of capital that is induced by the liquidity premium on intermediated claims.

The recommended inflation, however, does not come for free. To see this, combine (26), (27) and (28) to form:

$$
\mu=\beta L(q)\left(f^{\prime}(k)-\delta+\mu+s\right)
$$

By Lemma 3, we know that $\theta<\theta_{0}$ implies $q<q^{*}$ and $k>k^{*}$ under zero intervention $(\mu=1, s=0)$. Imagine for the moment that $s=0$. Then the recommendation $\mu(\theta)>1$ we just made has, by condition (43), the effect of reducing $k$, as desired. However, this creates a problem for AM sellers - how do we get them to produce the requisite amount of $\mathrm{AM}$ output, $q^{*}$, in return for a claim to PM output that is: 1) of low value to them and 2) increasing in number? The answer is to increase the dividend payment by collecting a fee and including it as part of the dividend.

This can be seen from the producer's FOC (18) which can be written as:

$$
w\left(k^{*}\right)=\frac{\phi_{2}}{\phi_{1}}+\frac{d}{\phi_{1}}=\frac{K^{*}(\theta)}{q^{*}}+\frac{D}{q^{*}}
$$

With $K^{*}(\theta)$ declining in $\theta$, to achieve the first-best, the aggregate dividend must increase as $\theta$ falls. This is achieved by charging a fee to collect the dividend and using this fee revenue to raise the dividend per share, thereby subsidizing its rate of return. This, in turn, pushes up the AM value of a ABM, which entices AM producers to supply $q^{*}$ despite the prospect of inflation. If a lump-sum tax instrument is available, the requisite fee revenue could be raised in this manner. But as we have shown above, lump-sum taxation is not necessary: one could design an incentive scheme that induces people to make voluntary contributions.

\section{Conclusion}

When commitment and record-keeping is limited, media of exchange are necessary to facilitate trade. Exactly what form these exchange media should take and how their supply should be managed over time remain open questions. In theory, a properly 
managed supply of fiat money may be sufficient but, as we show in this paper, fiat money is generally not necessary. The concept of monetary policy should be extended to include the management of intermediated exchange media. Our model remains silent on the question of whether the responsibility for the money supply should reside in the public or private sector. Because our results make no recourse to lumpsum taxation, our paper suggests that private intermediaries, like the central banks of ancient times, could in principle be left to manage the money supply. 


\section{References}

[1] Andolfatto, D. (2010). "Essential Interest-Bearing Money", Journal of Economic Theory 145, 1319-602.

[2] Aruoba, S.B. and R. Wright (2003). "Search, Money and Capital: A Neoclassical Dichotomy", Journal of Money, Credit and Banking 35, 1086-1105.

[3] Barro, R. (1979). "Money and the Price Level Under the Gold Standard", Economic Journal 89, 13-33.

[4] Fama, E. (1983). "Financial Intermediation and Price Level Control", Journal of Monetary Economics 12, 7-28.

[5] Fratianni, Michele (2006). "Government Debt, Reputation, and Creditors' Protection: The Tale of San Giorgio", Review of Finance, 10:(4): 487-506.

[6] Friedman (1969). "The Optimum Quantity of Money", in The Optimum Quantity of Money and Other Essays (Aldine. Chicago. IL) 1-50.

[7] Friedman, M. and A. Schwartz (1986). Has Government any Role in Money?", Journal of Monetary Economics 17 37-62.

[8] Geromichalos, A., J. Licari and J. Suarez Lledo (2007). "Asset Prices and Monetary Policy", Review of Economic Dynamics 10, 761-779.

[9] Hayek, F. (1976). "Denationalization of Money", (Institute of Economic Affairs, London). 1978, 2nd extended ed.

[10] Helpman, E. (1983). "Comments on 'Financial Intermediation and Price Level Control'", Journal of Monetary Economics 12, 29-31.

[11] Jacquet, N. and S. Tan (2012). "Money and Asset Prices with Uninsurable Risks," Journal of Monetary Economics 59, 784-797.

[12] King. R. (1983). "On the Economics of Private Money", Journal of Monetary Economics 12, 127-158.

[13] Klein, B. (1974). "The Competitive Supply of Money", Journal of Money, Credit, and Banking 6, 423-453.

[14] Klein, B. (1976), "Competing Monies", Journal of Money, Credit, and Banking 8, 513-519.

[15] Lagos, R. and G. Rocheteau (2008). "Money and Capital as Competing Media of Exchange" Journal of Economic Theory 142, 247-258. 
[16] Lagos, R. and R. Wright (2005). "A Unified Framework for Monetary Theory and Policy Analysis", Journal of Political Economy 113, 463-484.

[17] Lester B., A. Postlewaite, and R. Wright. (2012) "Information, Liquidity, Asset Prices and Monetary Policy", Review of Economic Studies 79, 1209-1238.

[18] Levine, David (1991). "Asset Trading Mechanisms and Expansionary Policy," Journal of Economic Theory, 54(1): 148-164.

[19] Molico, Miguel (2006). "The Distribution of Money and Prices in Search Equilibrium," International Economic Review, 47(3): 701-722.

[20] Rocheteau, G. (2011). "Payments and Liquidity under Adverse Selection", Journal of Monetary Economics 58, 191-205.

[21] Rocheteau, G. and R. Wright (2005). "Money in Search Equilibrium, in Competitive Equilibrium, and in Competitive Search Equilibrium", Econometrica 73, 175-202.

[22] Sargent, T. and N. Wallace (1983). "A Model of Commodity Money", Journal of Monetary Economics 2, 163-187.

[23] Wallace, N. (1983). "Legal Restrictions Theory of the Demand for 'Money' and the Role of Monetary Policy", Federal Reserve Bank of Minneapolis Quarterly Review/Winter.

[24] Waller, C. (2003). "Comment on 'Search, Money and Capital: A Neoclassical Dichotomy'", Journal of Money, Credit and Banking, 35(6, Part 2), 1111-17.

[25] Waller, C. (2011)."Random Matching and Money in the Neoclassical Growth Model: Some Analytical Results" Macroeconomic Dynamics 15(S2), 293-312. 